Boise State University

ScholarWorks

Geosciences Faculty Publications and

Presentations

Department of Geosciences

2-26-2014

\title{
Analyst A: Alternatives in Analysis of the UTexas1 Surface Wave Dataset
}

Paul Michaels

Boise State University

This is an author-produced, peer-reviewed version of this article. The final, definitive version of this document can be found online at Geo-Congress 2014: Technical Papers and Keynote Lectures, published by the American Society of Civil Engineers. Copyright restrictions may apply. doi: 10.1061/9780784413272.074 


\title{
Analyst A: Alternatives in Analysis of the UTexas1 Surface Wave Dataset
}

\author{
P. Michaels ${ }^{1}$, Member ASCE \\ ${ }^{1}$ Center for Geophysical Investigation of the Shallow Subsurface, Boise State Univer- \\ sity, 1910 University Drive, Boise, Idaho 83725-1535 , PH (208) 426-1929; FAX (208) \\ 426-1631; email: pm@cgiss.boisestate.edu
}

\begin{abstract}
In February of 2011 an earthquake event caused significant damage and loss of life in Christchurch, New Zealand. Such an event serves as motivation for improved foundation design and characterization of the shallow subsurface. In January of 2013, University of Texas engineers acquired surface wave data which has been made available to the ASCE GeoInstitute Geophysical Engineering Committee for a benchmark project. Participants were invited to process and interpret the common data set. This paper reports the results designated as those of "Analyst A". The active vibroseis and sledgehammer data were combined to produce a composite Rayleigh wave dispersion curve. Alternative soil profile representations were considered. The resulting image is of low velocity zone sandwiched between a faster surface zone and a faster profile down to 30 meters depth.
\end{abstract}

\section{INTRODUCTION}

The UTexas1 Surface Wave Dataset was acquired in Christchurch, New Zealand. The site was located approximately $1.5 \mathrm{~km}$ from the coast. The Geophysical Engineering Committee of the ASCE GeoInstitute has organized a benchmark project in which participating geophysical engineers have been invited to process and interpret this common data set with tools of their own choosing. The instructions were to work as much or as little of the data as each participant desired. The available data include both active-source and ambient noise sets. This participant has chosen to only work the active-source data.

The vibrator data were processed in the frequency domain, the hammer data in the time domain. The resulting dispersion curves were then combined to provide a composite dispersion curve. Inversion of the composite dispersion curve was done employing a 1-D-gradational soil profile in which the objects of the inversion are control points whose depth and shear-wave velocity assignment may vary. The reader is referred to Michaels (2011) for another example of this representation. 


\section{SELECTED DATA}

\section{Sledge Hammer Source}

The subset of the hammer data processed in this report are shown in Figure 1. The selection criteria included a desire to focus on the largest amplitude, fundamental mode surface waves. Thus, only near offsets (5-15 meters) were chosen. The further offsets would have included a refracted arrival, possible higher modes, and cultural noise, presumably due to traffic sourced beyond the furthest offset. The data are a vertical stack of 10 hammer blows. Data are vertical component signals, recorded on a 24-channel Geode seismograph with 24 bit ADC, $144 \mathrm{~dB}$ dynamic range, $90 \mathrm{~dB}$ anti-alias filter, 250 samples per second, 4 second recordings. The receivers were 4.5 $\mathrm{Hz}$ Geospace GS-11D geophones. The hammer was a $5.5 \mathrm{~kg}$ sledge, blows taken on a square aluminum strike-plate with overlying rubber pad. In the figure, each geophone channel has been rescaled by its norm to remove amplitude decay with offset and improve visibility of all the waveforms. While all 4 seconds of data were processed, only the first second of the recording are shown here.

Since reverse profiles were not available, a non-dipping interpretation of the apparent P-wave refraction and direct arrival was conducted. The results of that effort indicate a refractor with an apparent velocity of $1480 \mathrm{~m} / \mathrm{s}$ at a depth of approximately $12.7 \mathrm{~m}$. This is based on an estimate of $700 \mathrm{~m} / \mathrm{s}$ direct P-wave arrival velocity. A likely interpretation of this would be a refraction off the top of the water table. Since Rayleigh waves are only minimally affected by the P-wave velocity, and since reverse profiles were not available, a single value for the P-wave velocity profile was employed,

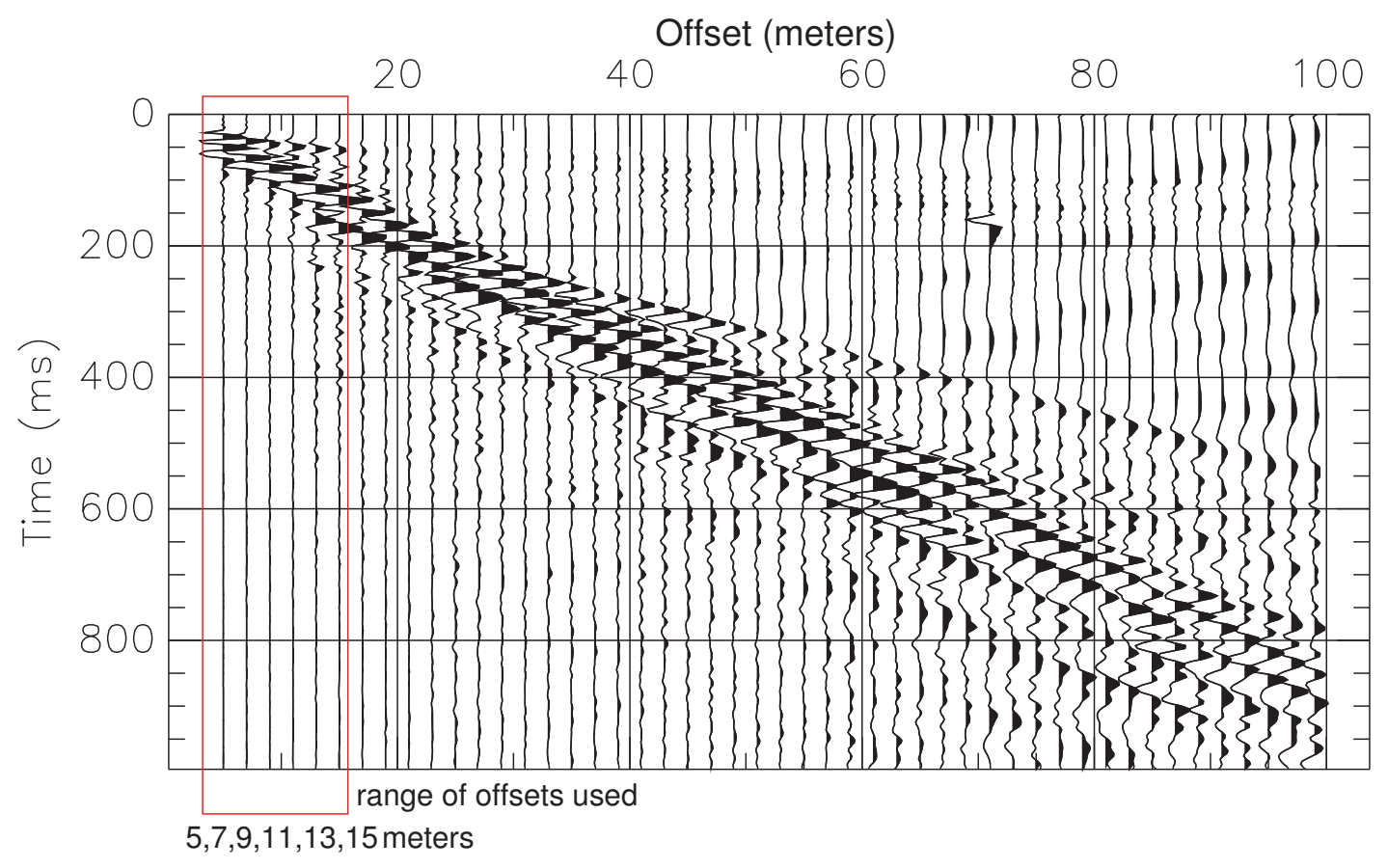

Figure 1. The vertical stack of the near source data was chosen to process. Only the first 6 channels were used in the analysis. 


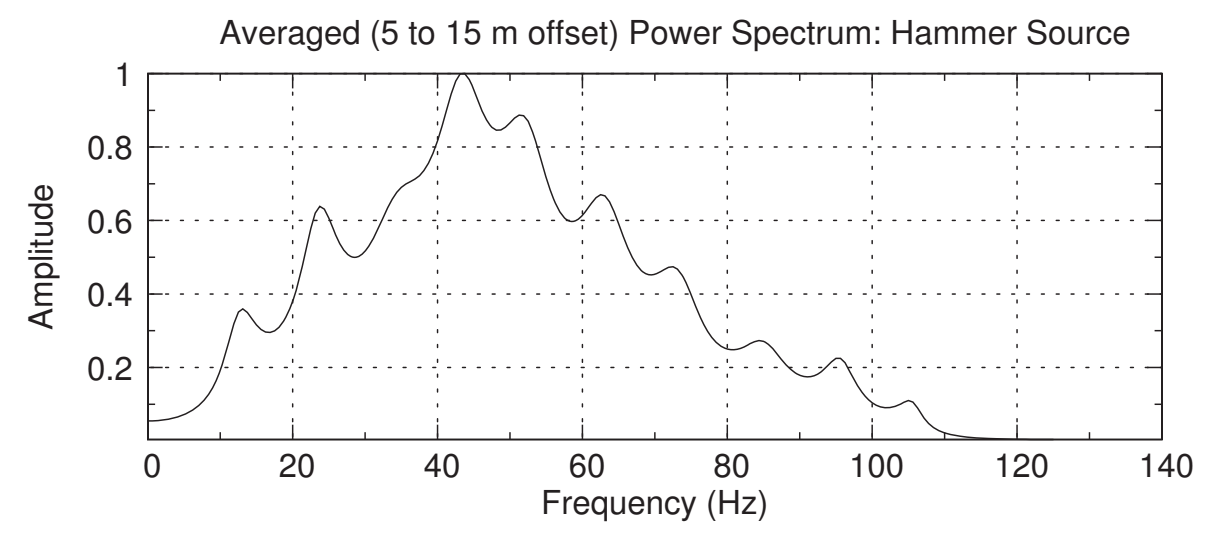

Figure 2. Average Yule-Walker all pole 20th order power spectrum estimate. This represents the maximum range of frequencies available. However, one can not assume all amplitudes represent Rayleigh waves exclusively.

along with a single value for mass density (also a minor influence on Rayleigh waves). As a check, an alternative model employing the P-wave refraction solution was found to make no difference on the computed dispersion.

To focus on the fundamental mode Rayleigh wave, only the first 6 channels were employed in the analysis. An estimate of the hammer data power spectrum was computed from the average of the chosen 6 signal auto correlations using a Yule-Walker 20th order all pole data model (Karl, 1989). This is shown in Figure 2. At the low end, the signal strength below about $10 \mathrm{~Hz}$ becomes unusable. At the high end, additional processing found that higher modes began to appear above $35 \mathrm{~Hz}$. In addition, amplitudes represent a mixture of wave types, and may include body waves, particularly at high frequencies.

\section{DISPERSION AND TIME DOMAIN ANALYSIS, HAMMER SOURCE}

The data were processed for dispersion by first applying narrow band $(0.25 \mathrm{~Hz})$ filters in the time domain. The spectrum from 5 to $50 \mathrm{~Hz}$ was filtered in $0.25 \mathrm{~Hz}$ steps. Each filtered version was then subjected to beam steering and stacking with a set of 80 trial velocities ranging from 100 to $400 \mathrm{~m} / \mathrm{s}$. A semblance value was computed for each trial alignment, and a Golden Section search was executed to find the maximum semblance. Semblance is a scalar measure of coherence. Semblance was computed as a value for the entire 4.0 second record length:

$$
S=\frac{1}{M} \cdot \frac{\sum_{j=1}^{N}\left(\sum_{k=1}^{M} u_{k j}\right)^{2}}{\sum_{j=1}^{N} \sum_{k=1}^{M}\left(u_{k j}^{2}\right)}
$$

where there are $M$ signals and $N$ samples per signal. The $j t h$ sample in the $k t h$ signal is given by $u_{k j}$. When the data are aligned with a velocity that maximizes semblance, there will remain signal to signal misalignments along the alignment trend. These are used to determine confidence limits for the determined phase velocity. 
(A) Hammer Source Offsets 5 to $15 \mathrm{~m}$

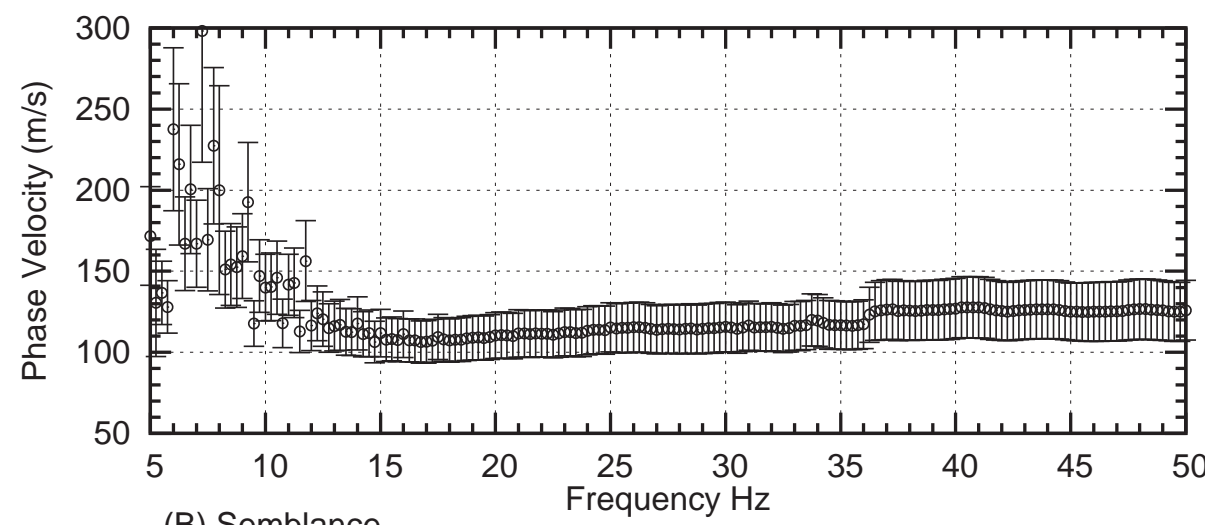

(B) Semblance

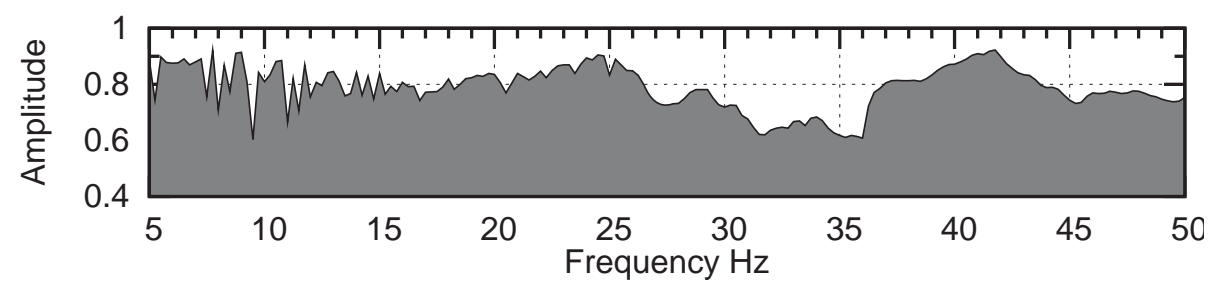

Figure 3. (A) Dispersion curve (with $95 \%$ confidence limits) from semblance analysis, and $(B)$ Semblance values for determined phase velocities. Note that the uncertainties increase significantly below $12 \mathrm{~Hz}$. This is due to the loss of signal strength (see Figure 2). The discontinuity at about $36 \mathrm{~Hz}$ may be due to higher mode interference.

Confidence Limits At each frequency, temporal cross-correlations in the time domain are used to compute relative time shift misalignments with respect to a reference signal (first signal in the set). The angle between the $k t h$ signal, $u_{k}$, and the reference signal $u_{1}$ is computed as

$$
\theta_{k}=\cos ^{-1} \frac{\left.\left(\sum_{j=1}^{N}\left(u_{1 j}\right) \cdot u_{k j}\right)\right)}{\left\|u_{1}\right\| \cdot\left\|u_{k}\right\|}
$$

where $\|u\|$ is the L2 norm of a signal. This angle between filtered signals resolves to a time shift given by,

$$
\Delta t_{k}=\frac{\theta_{k}}{2 \pi f}
$$

where $f$ is the frequency in $\mathrm{Hz}$. These $\Delta t_{k}$ values are treated as though they were the residuals in a least squares linear fit of offset, $x_{k}$, and arrival time, $t_{k}$,

$$
\left[\begin{array}{cc}
x_{1} & 1 \\
x_{2} & 1 \\
\vdots & \vdots \\
x_{6} & 1
\end{array}\right] \cdot\left[\begin{array}{c}
m \\
b
\end{array}\right]=\left[\begin{array}{c}
t_{1} \\
t_{2} \\
\vdots \\
t_{6}
\end{array}\right]
$$

where $m=1 / V$ is the slope (slowness) and $b$ an intercept. Of course, there are no time picks, $t_{k}$, when considering phase velocity. We can synthesize these from the ratio of 
source-receiver offset divided by the solution phase velocity. We only care about the uncertainty of the slowness as estimated from the signal to signal misalignment, $\Delta t_{k}$. We compute the variance, $\sigma_{t}^{2}$, of the $\Delta t_{k}$ about the mean $\Delta t$, in the usual way. The estimate of the standard deviation in velocity is ultimately found to be:

$$
\pm \sigma_{v}= \pm \sigma_{t} \sqrt{\left(\frac{M\left(M \sum x^{2}-\left(\sum x\right)^{2}\right)^{3}}{\left(\sum x \sum t-M \sum x t\right)^{4}}\right)}
$$

This standard deviation is scaled $\left( \pm \sigma_{v} \times 2.4469\right)$ for a six degree of freedom Student-T distribution to compute $95 \%$ confidence error bars shown in Figure 3.

\section{VIBROSEIS DATA}

The data were recorded on 21 vertical component $1.0 \mathrm{~Hz}$ geophones (Mark Products L-4C), gravity coupled to an aluminum leveling base which was in turn coupled to the ground with $7.6 \mathrm{~cm}$ spikes. Geophones were space 10 meters apart. The source was a large triaxial vibroseis truck from the University of Texas, known as TRex. The recording system consisted of two 16 channel Mobilyzer dynamic signal analyzers manufactured by Data Physics Corporation (24 bit ADC, 120 dB dynamic range and $110 \mathrm{~dB}$ anti-alias filters). Only the near source data set was chosen for final processing. The source to near geophone offset was 20 meters. The minimum sample rate was 25.6 samples per second. The length of the recording varied as the vibrator acquired data at constant frequencies which were conducted from 10 to $1 \mathrm{~Hz}$ in $0.1 \mathrm{~Hz}$ steps. A minimum of 50 cycles were recorded and averaged in the frequency domain. Analysts were provided the complex spectra to work with.

\section{DISPERSION AND FREQUENCY DOMAIN ANALYSIS, VIBROSEIS SOURCE}

In the frequency domain, adjacent pairs of signals were effectively cross correlated by complex multiplication of one signal with the complex conjugate of the other signal. The results were viewed as amplitude and phase difference. The amplitude of the product of signal pairs provided cross-power spectra. The phase difference was used to compute the phase velocity. Thus, the phase velocity in meters/second for any frequency was computed as:

$$
V=\frac{2 \pi f \cdot \Delta x}{\Delta \phi}
$$

where $\Delta \phi$ is the unwrapped phase difference in radians, $\Delta x=10$ meters, the geophone spacing, and $f$ is the frequency in $\mathrm{Hz}$. A total of 20 pairs were evaluated for all geophones to permit computation of uncertainties and error bars. Figure 4 shows the results. Note that the scatter in phase velocities increases at frequencies below 5 $\mathrm{Hz}$, and that is the point where the cross spectra also become quite low in amplitude (indicating a failure to generate signals at lower frequencies). It appears that adequate signal was recorded above $5 \mathrm{~Hz}$, as those results show far less scatter. At each 0.1 $\mathrm{Hz}$ frequency step, the average and sample variance was computed to generate the low 
(A) Vibroseis Dispersion

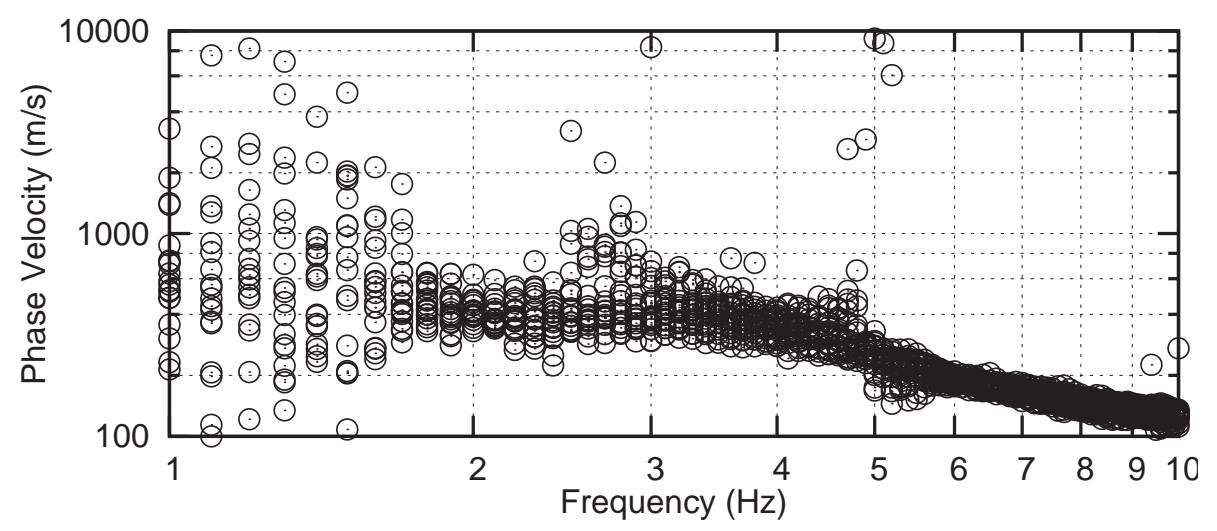

(B) Cross Power Spectrum

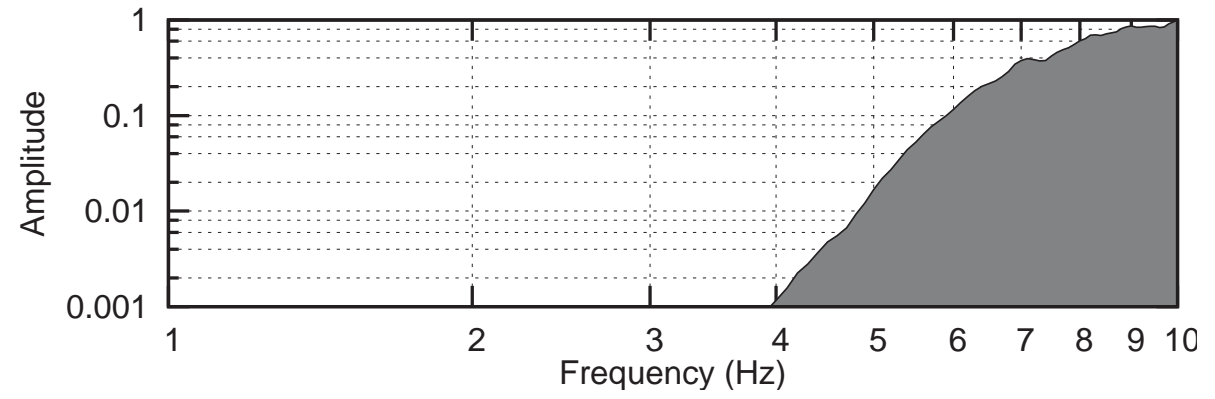

Figure 4. (A). The estimates of phase velocity dispersion for 20 pairs of geophones. (B). The cross power spectrum shows a marked limit to low frequency content. Thus, there is an increase in scatter of the dispersion estimates which maps to larger uncertainties and error bars at low frequencies.

frequency portion of the dispersion curve with error bars. Error bars for $95 \%$ confidence were estimated by scaling the sample standard deviation by 2.086 (Student-T, 20 degrees freedom).

Figure 5 shows the dispersion with mean values plotted as circles with error bars computed from the scatter around the mean as shown in Figure 4. Since the signal vanishes below about $5 \mathrm{~Hz}$, only the 5 to $10 \mathrm{~Hz}$ band is shown in Figure 5 .

\section{MERGING VIBROSEIS AND HAMMER DISPERSION}

The final dispersion curve was fabricated from the two sources. The vibroseis dispersion provided the needed low frequency data which sense the deeper regions of the soil profile. The hammer data sense the very near surface. The composite dispersion is shown in Figure 6. The error bars present two different confidence conditions. The vibroseis (5 to $10 \mathrm{~Hz}$ ) confidence limits are based on 20 pairs of cross-correlations conducted in the frequency domain. The hammer source confidence limits are based on 6 signal misalignments at the phase velocity which maximizes semblance. The Student T-distribution was used to estimate the $95 \%$ confidence bounds. 
(A) Christchurch New Zealand Vibroseis Source $=-20 \mathrm{~m}$

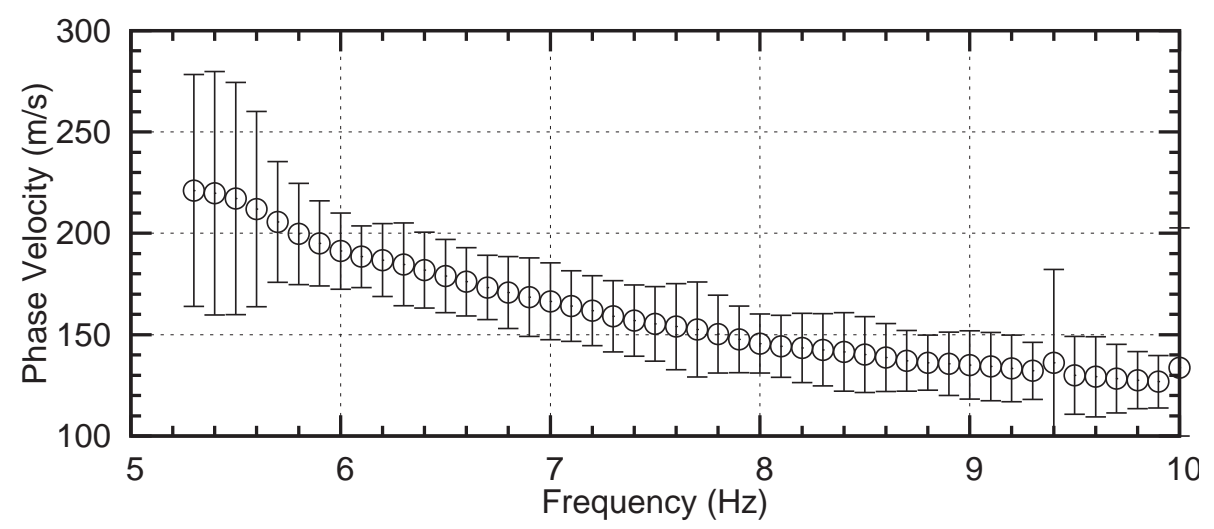

(B) Cross Power Spectra

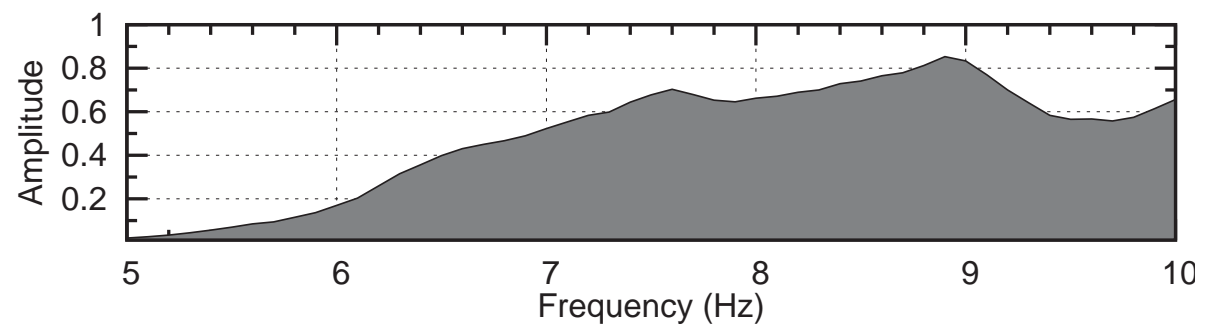

Figure 5. (A). The mean dispersion for the vibroseis data with $95 \%$ confidence limits. (B). Cross power spectrum for the 5 to $10 \mathrm{~Hz}$ band.

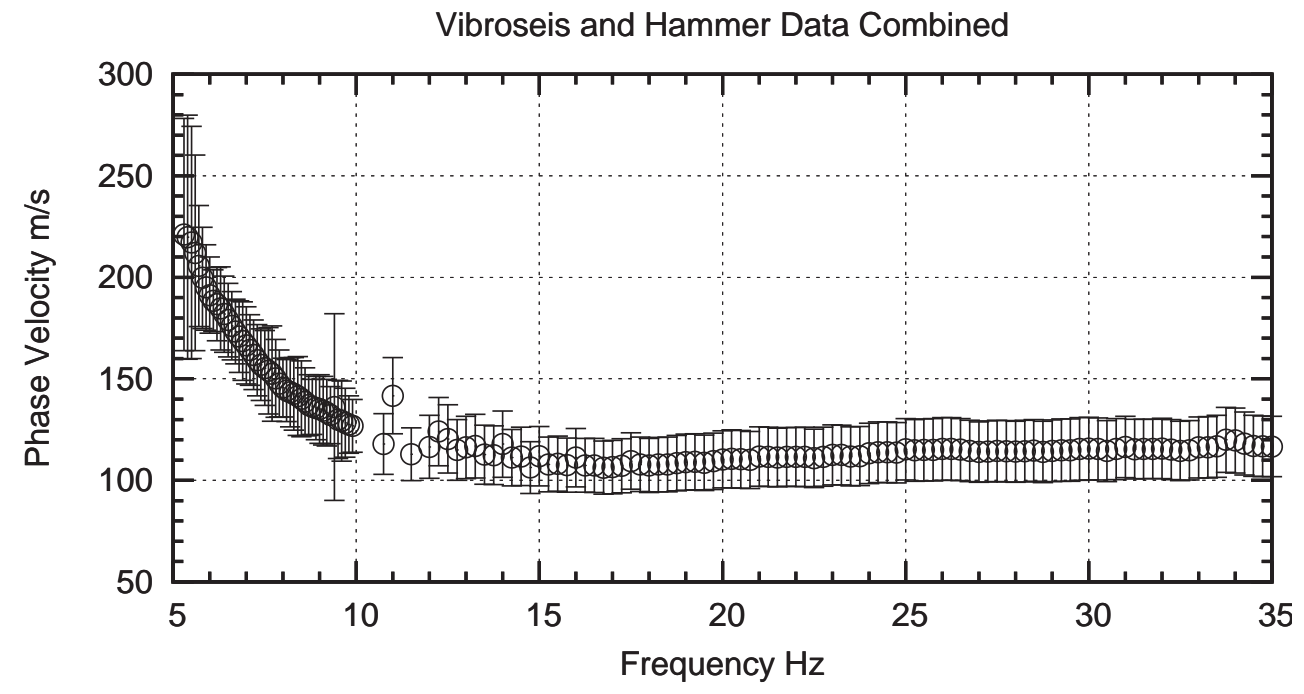

Figure 6. Final dispersion curve is the concatenation of the vibroseis and hammer curves. Error bars are for $95 \%$ confidence using the Student-T distribution. 


\section{INVERSION OF MERGED DISPERSION}

The dispersion curve of Figure 6 was inverted using a soil representation that held the mass density and P-wave velocity constant, while varying the S-wave velocity. The $S$-wave velocity was specified by control points. Each control point was an object of the inversion. Control points define a depth and S-velocity pair. Between the control, the elastic shear modulus (not velocity) varies linearly in 0.2 meter stepwise layers. Thus, confidence estimates of the solution involve two degrees of freedom (depth and S-velocity), each with their own error bars. The deepest control point was held fixed (both in S-velocity and depth). The surface control point was held constant in depth, but allowed to change in S-velocity. For Rayleigh wave data, sensitivity to P-wave velocity and density is low, so these were held constant. The resulting fundamental mode solution is tabulated in Table 1. The top and bottom control point depths are fixed, but the velocities at those points are a result of the inversion.

Table 1. Inversion Results with 95\% Confidence Limits

\begin{tabular}{rrrr}
\hline Depth $(\mathrm{m})$ & $V_{s}(\mathrm{~m} / \mathrm{s})$ & $V_{p}(\mathrm{~m} / \mathrm{s})$ & $\rho\left(\mathrm{kg} / \mathrm{m}^{3}\right)$ \\
\hline $0 \pm 0.000$ & $209.8 \pm 38.2$ & 1700. & 2169. \\
$1.13 \pm 0.2$ & $97.9 \pm 3.5$ & 1700. & 2169. \\
$2.11 \pm 0.5$ & $88.0 \pm 1.4$ & 1700. & 2169. \\
$6.45 \pm 0.5$ & $155.8 \pm 5.3$ & 1700. & 2169. \\
$13.58 \pm 0.5$ & $207.1 \pm 5.6$ & 1700. & 2169. \\
$30.00 \pm 0.000$ & $450.0 \pm 165.5$ & 1700. & 2169. \\
\hline
\end{tabular}

The inversion was computed using singular value decomposition, truncating the singular values used to the six largest. Truncation of singular values is used to regularize a solution (Menke, 1989). The representation shown in Table 1 was used to compute calculated dispersion for comparison with the observed measurements of dispersion. The uncertainties in parameters were computed from the dispersion data covariance matrix, $C_{d}$, by

$$
C_{m}=H \cdot C_{d} \cdot H^{T},
$$

where $C_{d}$ is a diagonal matrix with the data variance determined for the measured dispersion on the diagonal. The parameter variance is found on the diagonal of matrix $C_{m}$, and $H$ is the ordinary least squares inverse computed for the selected singular values (Menke, 1989).

Further, a limiting upper and lower bound (fast and slow velocity profile) representation was computed from the $95 \%$ confidence limits. The slow case added the error bars to depth, and subtracted the error bar from velocity. The limiting fast case did just the opposite, subtracting error bars from the solution depth control points, and adding the error bars to the velocity control. The dispersion for those limiting cases is also shown with that for the solution in Figure 7.

The S-wave soil profile with 0.2 meter layers was used in computing the dispersion shown in Figure 7. Figure 8 shows the soil profile by plotting both the control and 
the interpolated layers between control points that computed the dispersion for comparison with the observed. Also shown in the figure are the limiting slow and fast cases which represent $95 \%$ confidence limits. The dispersion shows a minimum Rayleigh wave velocity around $17 \mathrm{~Hz}$, and then rises at higher frequencies. This trend corresponds to the velocity profile minimum at 0.211 meters in the control (see Table 1). This suggests a soft layer sandwiched between a stiffer surface layer and the deeper soils. The vibrator data was found to be essential in sensing S-velocity down to 30 meters. Without that contribution, the hammer source alone would be insufficient.

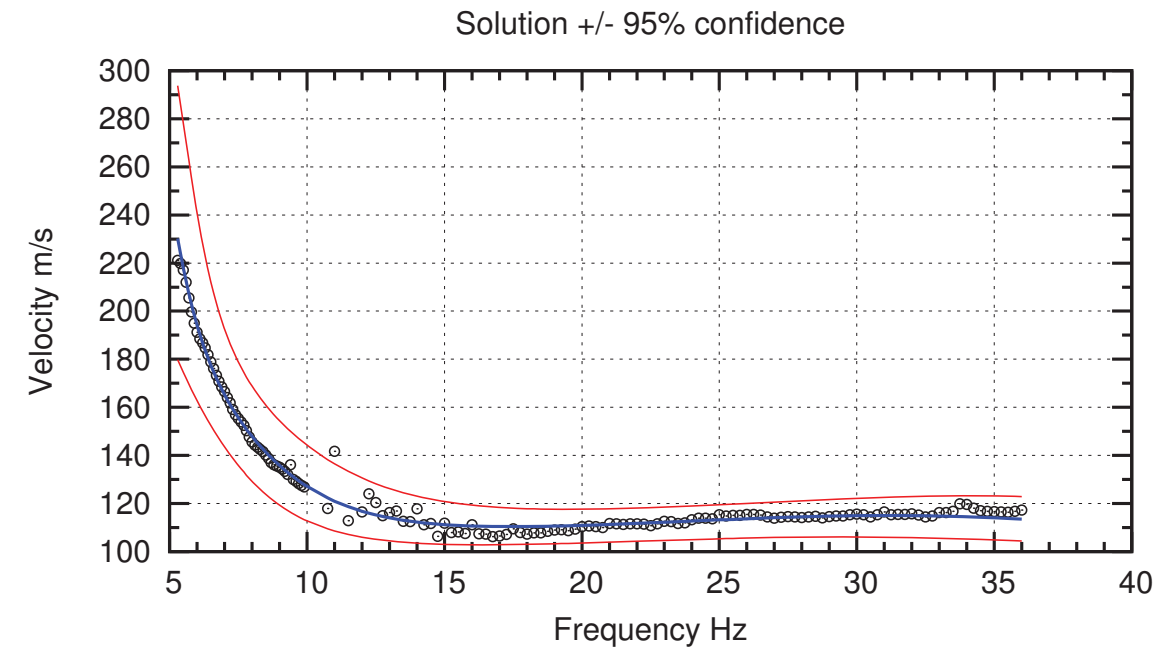

Figure 7. Calculated dispersion from Table 1 soil profile solution and the limiting "fast" and "slow" cases with observed dispersion shown as circles.

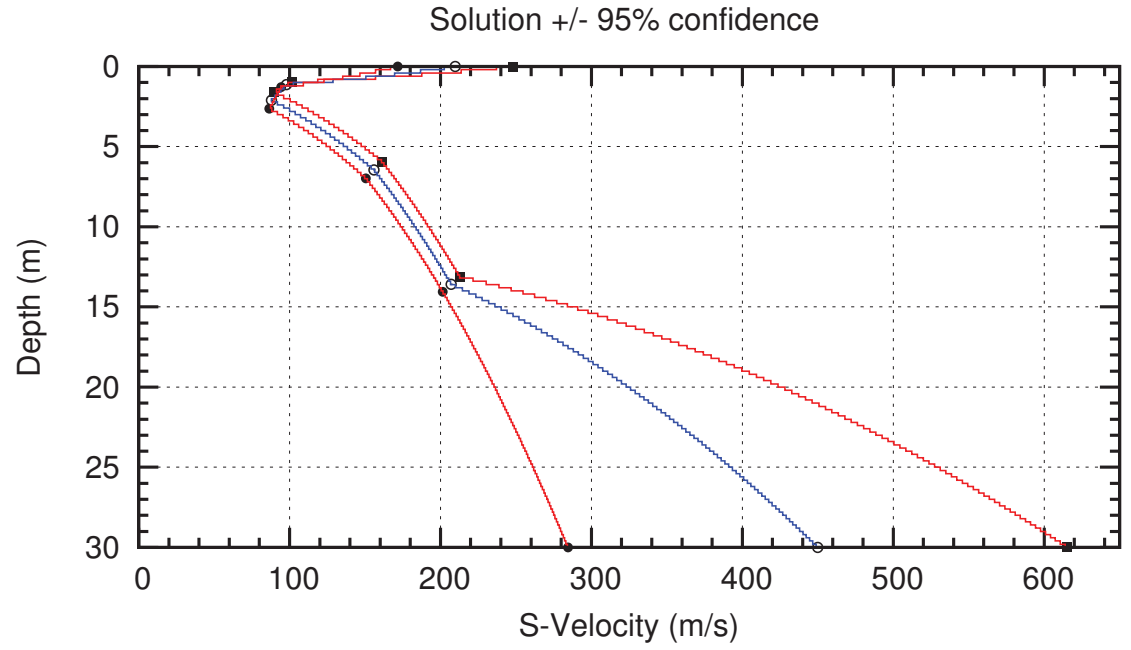

Figure 8. Soil profile of shear velocity with "fast" and "slow" limiting cases corresponding to the $95 \%$ confidence limits. Shear modulus (not shear velocity) is linearly interpolated in 0.2 meter layer steps between control points. 
This is an author-produced, peer-reviewed version of this article. The final, definitive version of this document can be found online at Geo-Congress 2014: Technical Papers and Keynote Lectures, published by the American Society of Civil Engineers. Copyright restrictions may apply. doi: 10.1061/9780784413272.074

\section{COMPUTING VS30}

Of interest to earthquake engineering is the problem of estimating the shearwave velocity profile in the first 30 meters. Research on characteristics important to earthquake engineering have resulted in formulas for near surface shear wave velocity being added to building codes (Dobry et al. , 2000). Current building codes refer to this as $V s_{30}$. It is computed for a layered profile by formula 36-1 of UBC-97, for example.

$$
V S_{30}=\frac{\sum Z_{i}}{\sum\left(Z_{i} / V s_{i}\right)}
$$

Uncertainties found in this analysis of measured dispersion do not affect $V s_{30}$ enough to change the soil profile type which is SD, stiff soil. The definition of stiff soil is $\left[180<V s_{30}<360\right] \mathrm{m} / \mathrm{s}$. Table 2 summarizes the results of the computation for the solution and the two limiting cases.

Table 2. Shear velocity, $V S_{30}$, from alternative soil profiles.

\begin{tabular}{ll}
\hline Case & $V S_{30}$ \\
\hline slow & $180 . \mathrm{m} / \mathrm{s}$ \\
solution & $210 . \mathrm{m} / \mathrm{s}$ \\
fast & $238 . \mathrm{m} / \mathrm{s}$ \\
\hline
\end{tabular}

\section{SOLUTION APPRAISAL}

The maximum depth sensed by the surface waves is limited by the low frequency content of the source. In this case, the lowest frequency the vibrator shook ground was $1 \mathrm{~Hz}$. In retrospect, it appears that this intention exceeded the capacity of the hardware to produce Rayleigh waves at that low frequency. The scatter in the dispersion estimates shown in Figure 4 suggest that the lowest frequency Rayleigh waves actually generated were around 5 to $6 \mathrm{~Hz}$. Some scatter might be expected due to lateral soil variation, but the cross-power spectral amplitudes are interpreted as a loss of signal below $5 \mathrm{~Hz}$. As can be seen by the displacements computed from the final solution in Figure 9, the motion significantly diminishes below 15 meters. This conclusion is in agreement with the widening of the confidence intervals as shown in Figure 8.

Then there is the question of higher modes. Could those be used to extend the depth of exploration? One of the dilemmas in selecting a range of offsets to analyze is the appearance of higher modes with increased offsets. In selecting only the first 6 signals closest to the source, we reduce the influence of higher modes. In trials processing 10 meter intervals at increasing offset, the author found higher modes to interfere with the fundamental. Figure 10 shows the first nine modes of surface waves for the solution based on the fundamental mode inversion. The vibrator dispersion is shown as circles, the hammer dispersion as triangles. Note that the solution fundamental mode is a good fit to the observed dispersion. However, at around $35 \mathrm{~Hz}$, the fundamental mode pinches close to the first higher mode. This raises the possibility that a semblance maximum might jump from the fundamental to the first mode. If one were to interpret 
Fundamental Motion at $6 \mathrm{~Hz}$

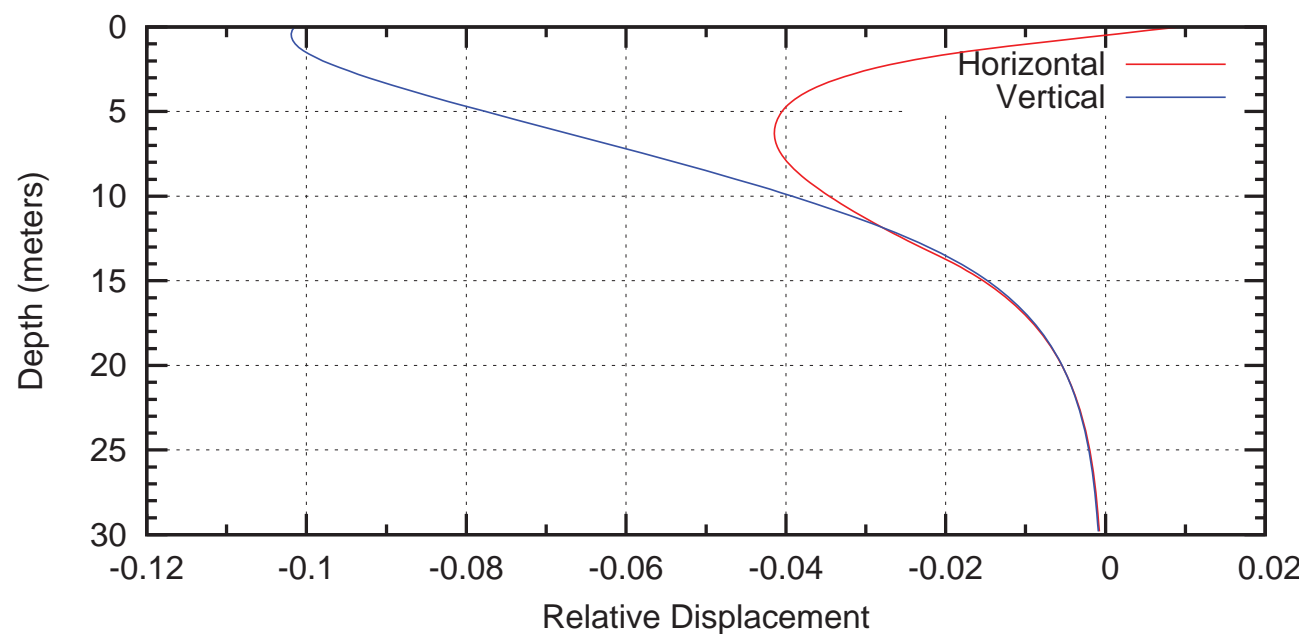

Figure 9. The motion of the fundamental mode Rayleigh wave at $6 \mathrm{~Hz}$ does not appear to sense the subsurface much beyond 15 meters depth.

the first mode as part of the fundamental curve, it would lead to errors in the solution. Tests on larger offset ranges resulted in the velocity scan actually jumping from the fundamental to the higher modes. It is for that reason that the near offsets were selected for measuring dispersion. The real challenge for inversions employing higher modes is to correctly identify the modes and keep them straight.

\section{SUMMARY}

There are a number of compromises needed in selecting a portion of a data set to analyze. On the one hand, one would like to make measurements over a wide range of offsets to reduce uncertainties in the measured dispersion. But as the range of offsets increases, a 1-D layered analysis of surface waves becomes inappropriate in the context of possible geologic variation in the soil profile over an increasing range of observation. Further, if phase velocity is inverted, as is the case here, there is no temporal resolution. All the different wave fields possible enter into the analysis. This may include nonsurface waves (like refractions and reflections) and higher mode surface waves.

When one examines Figure 10, it is apparent that the solution fits the data well for those frequencies sampled. The lack of low frequency content below $5 \mathrm{~Hz}$ revealed that the deepest extent of the solution is not well determined. That observation is reinforced by the motion plotted in Figure 9.

However, one can also analyze signals too close to the source. The expanding wave field needs to reach to sufficient depth and interact with the soil profile of interest. One also prefers to be in the far field. These factors are hard to assess before anything is known about the soil profile. Thus, the collection of a wide range of offsets permits an analyst to probe and select that subset of the data most appropriate to the problem at hand. This data set provided that opportunity. While only 6 near offsets were selected in this case, that result should not be generalized. 
This is an author-produced, peer-reviewed version of this article. The final, definitive version of this document can be found online at Geo-Congress 2014: Technical Papers and Keynote Lectures, published by the American Society of Civil Engineers. Copyright restrictions may apply. doi: 10.1061/9780784413272.074

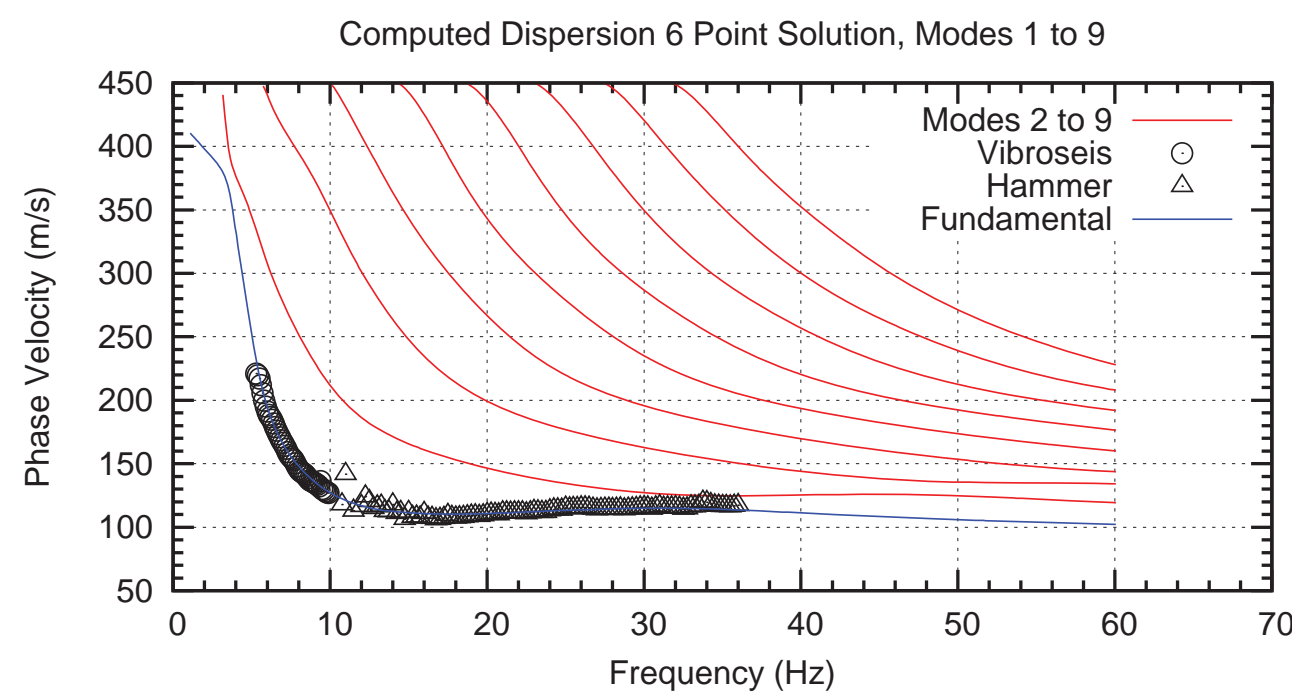

Figure 10. Note that the solution matches the fundamental mode well, but around $35 \mathrm{~Hz}$ the fundamental and first higher mode pinch close together, making mode identification difficult when computing dispersion curves.

\section{ACKNOWLEDGMENTS}

The author wishes to thank the Dr. Brady Cox for the collected data and organization of this project for the Geophysical Engineering Committee of the ASCE GeoInstitute. The National Science Foundation (NSF) provided support for collection of the UTexas1 Surface Wave Dataset through a RAPID award to Brady R. Cox at University of Texas under grant CMMI-1303595 (RAPID: Deep Shear Wave Velocity Profiling for Seismic Characterization of Christchurch, NZ - Reliably Merging Large Active-Source and Passive-Wavefield Surface Wave Methods). Any opinions, findings, and conclusions or recommendations expressed in this paper are those of the author and do not necessarily reflect the views of the NSF

\section{REFERENCES}

Dobry, R., Borcherdt, R. D., Crouse, C. B., Idriss, I. M., Joyner, W. B., Martin, G. R., Power, M. S., Rinne, E. E., \& Seed, R. B. (2000). New Site Coefficients and Site Classification System Used in Recent Building Seismic Code Provisions. Earthquake Spectra, 16(1), 41-67.

Karl, J. H. (1989). An Introduction to Digital Signal Processing. Academic Press.

Menke, W. (1989). Geophysical data analysis, discrete inverse theory. Academic Press. San Diego 289pgs.

Michaels, P. (2011). Establishing Confidence in Surface Wave Determined Soil Profiles. In: Juang, C. Hsein, Phoon, Kok Kwang, Puppala, Anand J., Green, Russell A., \& Fenton, Gordon A. (eds), GeoRisk 2011 Geotechnical Risk Assessment and Management. GSP 224, no. 837. GeoIstitute of ASCE. 